\title{
Valve Concepts for Microfluidic Cell Handling
}

\author{
M. Grabowski, A. Buchenauer
}

\begin{abstract}
In this paper we present various pneumatically actuated microfluidic valves to enable user-defined fluid management within a microfluidic chip. To identify a feasible valve design, certain valve concepts are simulated in ANSYS to investigate the pressure dependent opening and closing characteristics of each design. The results are verified in a series of tests. Both the microfluidic layer and the pneumatic layer are realized by means of soft-lithographic techniques. In this way, a network of channels is fabricated in photoresist as a molding master. By casting these masters with PDMS (polydimethylsiloxane) we get polymeric replicas containing the channel network. After a plasma-enhanced bonding process, the two layers are irreversibly bonded to each other. The bonding is tight for pressures up to 2 bar. The valves are integrated into a microfluidic cell handling system that is designed to manipulate cells in the presence of a liquid reagent (e.g. PEG - polyethylene glycol, for cell fusion). For this purpose a user-defined fluid management system is developed. The first test series with human cell lines show that the microfluidic chip is suitable for accumulating cells within a reaction chamber, where they can be flushed by a liquid medium.
\end{abstract}

Keywords: microfluidic chip, valve, cell handling, simulation, pneumatic actuation, polydimethylsiloxane.

\section{Introduction}

The integration of new characteristics into a cellular system is an essential challenge in modern biotechnology. Methods which are suitable for fusing different cell types to generate hybrid cells which show hybrid characteristics of the two original cells are of central interest in this area. The hybridoma technology, for example, generates hybrid cell lines by fusing antibodyproducing B-cells with a cancerous myeloma cell line. As a result of this fusion, the generated hybridomas produce monoclonal antibodies. Cells can be fused for example chemically in the presence of polyethylene glycol (PEG), electrically by the application of pulsed electric fields (electrofusion), or by means of a focused laser beam (laser-induced fusion). Fusion is traditionally performed in a cell bulk of two mixed cell lines. The drawback of this traditional type of cell fusion within a bulk is the statistical combination of the cells. Equal concentrations in both cell suspensions will lead to $50 \%$ heterokaryon formation (fusion of two different cell types) and $50 \%$ monokaryon formation (fusion of cells of the same type). User-controlled and observable cell fusion is not realizable with this method. In recent years, the use of microfluidic systems has become an eligible method for applications like biochemical assays, medical diagnostics, drug delivery, cell sorting and cell manipulation, as they enable transportation, isolation and manipulation of small amounts of liquids and cells or even single cells. This microfluidic system enables the user to select certain cells which should be manipulated or investigated. Polydimethylsiloxane (PDMS) is a well suited material for these applications, as microfluidic systems can be developed very cheaply and rapidly by methods of soft lithography $[1,2]$.

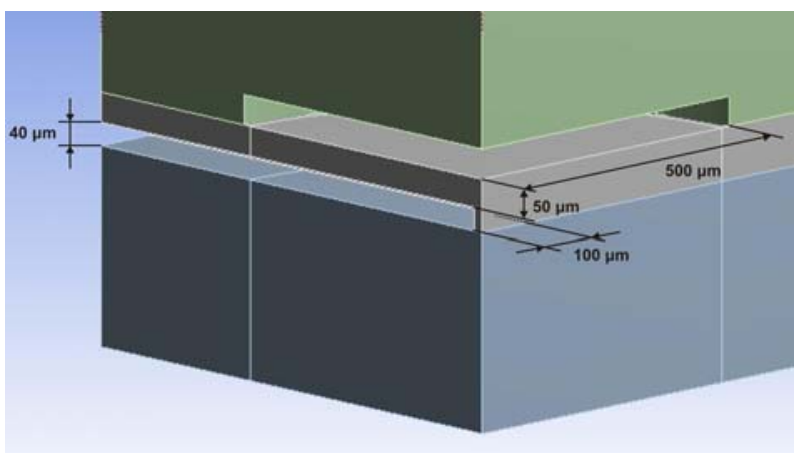

Fig. 1: Common geometrical parameters of all valve types

\section{Materials and methods}

\subsection{Valve design}

Various valve concepts were designed and fabricated in PDMS using soft-lithographic techniques to find a feasible geometry. The geometry of the pneumatic layer is equal in all investigated valve types, as is the width and height of the fluidic channel. The changes are only in the form of the fluidic channel (rectangular or rounded) and the order of the two functional layers (Fig. 2, 3, 4, 5). Figure 1 shows the general geometrical parameters of all investigated valve types. The pneumatic chamber has a diameter of $1 \mathrm{~mm}$. The height of the chamber is $70 \mu \mathrm{m}$. A change in height has no influence on the characteristics of the valve, as long as it is high enough to make upward bending of the membrane possible. The cross-section of the fluidic channel is $200 \mu \mathrm{m} \times 40 \mu \mathrm{m}$. The membrane is $50 \mu \mathrm{m}$ in thickness. The first valve geometry (valve type 1) was built up with an interrupted microfluidic chan- 
nel and a pneumatic chamber above the interruption (Fig. 2) similar to [3, 4]. The bar is $30 \mu \mathrm{m}$ in width.

This valve was at first operated passively, i.e. no pressure (positive or negative) was applied to the pneumatic layer. With no pressure difference between the pneumatic layer and the fluidic layer, the bar blocks the flow and the valve remains closed. Therefore valve type 1 is intrinsically closed. When pressure is applied to the filled microfluidic channel, the membrane around the interruption lifts up and the fluid is able to pass the valve. This is the open state of the valve. To optimize this concept the valve was operated actively by applying a positive or negative pressure onto the pneumatic chamber. The second valve geometry (valve type 2) was designed without the intersection in the fluidic channel, using a microfluidic channel with just a rectangular cross-section (Fig. 3).

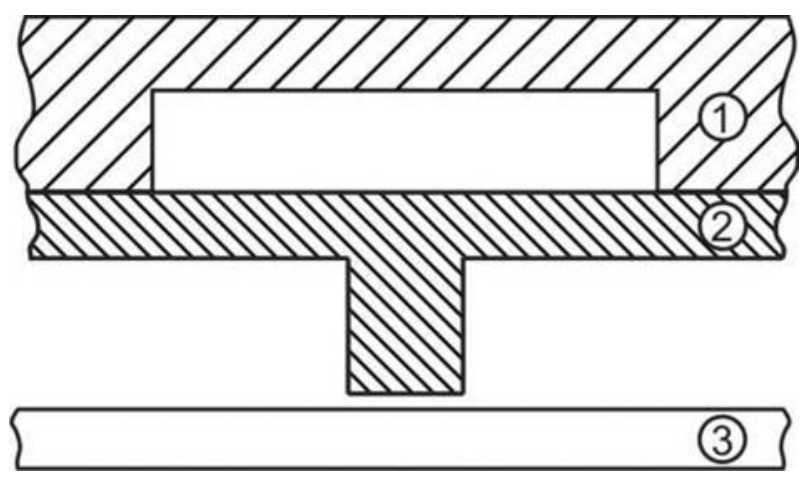

Fig. 2: Valve type 1. Cross-section of valve area with interrupted fluidic channel. 1: pneumatic layer; 2: fluidic layer; 3: substrate

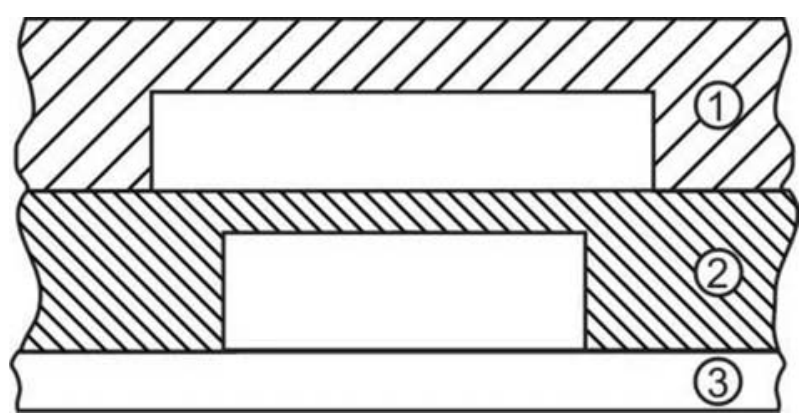

Fig. 3: Valve type 2. Cross-section of valve area with rectangular channel geometry. 1: pneumatic layer; 2: fluidic layer; 3: substrate

This valve type can only work when actively actuated, because it is intrinsically open. To avoid the disadvantages of valve type 2 , which are explained in detail in the results, we designed a subtype of this geometry. This optimized valve type 3 is characterized by a fluidic channel with a rounded cross-section (Fig. 4). The rounded geometry of the fluidic channel is realized by heating the photoresist master to $140{ }^{\circ} \mathrm{C}$ for 5 minutes, similar to [5]. For further optimization based on the ANSYS simulations, we designed a valve type 4 similar to type 3 . It is also characterized by a rounded fluidic cross-section. The significant difference is the reverse order of the PDMS layers (Fig. 5). The pneumatic layer is now below the fluidic channel.

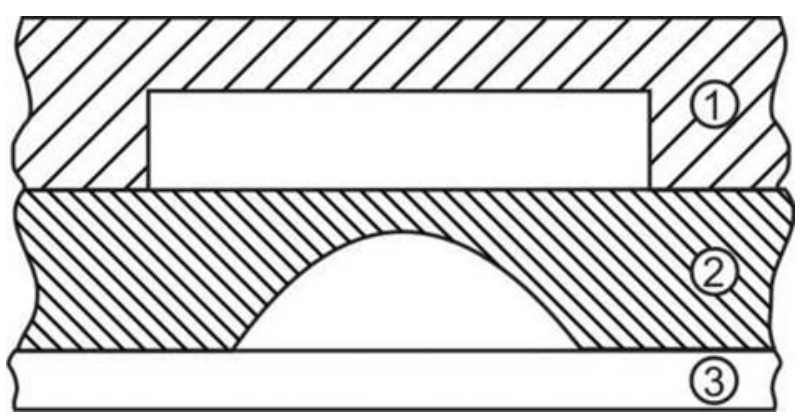

Fig. 4: Valve type 3. Cross-section of valve area with round channel geometry. 1: pneumatic layer; 2: fluidic layer; 3 : substrate

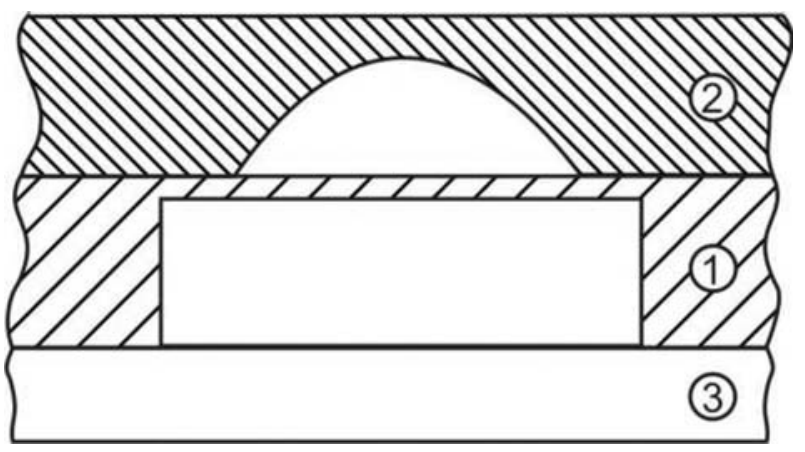

Fig. 5: Valve type 4. Cross-section of valve area with round channel geometry. 1: pneumatic layer; 2: fluidic layer; 3: substrate

\subsection{Fabrication}

The fabrication of the microfluidic chip starts with the design of a microfluidic and pneumatic channel system using CAD software. This layout is then printed in $25 \mathrm{kdpi}$ to a transparency which acts as a photomask in photolithography to structure AZ nLof / AZ 9260 photoresist (AZ Electronic Materials) on a silicon wafer. By casting liquid polymer onto the master wafer the channel network is transferred to the PDMS device. The PDMS (Sylgard 184, Dow Corning) is a twocomponent system consisting of a base and a curing agent, which are mixed in a ratio of 10:1 (base:curing agent). Subsequently, the liquid pre-polymer is poured onto the master and is cured in a convection oven at $80^{\circ} \mathrm{C}$ for 20 minutes. Afterwards, the PDMS replica can be peeled off from the wafer. The channel system can be made accessible by punching out holes into the PDMS slide using a hollow needle. The cast PDMS layers can be bonded in two ways. Bringing the polymer substrates into tight contact without a pretreatment leads to reversible bonding of the two slabs due to van der Waals forces $[1,2]$. Because this kind of 
bonding does not withstand elevated pressures $>0.4$ bar in the channel network, we prefer plasma enhanced bonding of the substrates. If the PDMS devices are exposed to an $\mathrm{O}_{2}$-plasma, covalent $\mathrm{Si}-\mathrm{O}-\mathrm{Si}$ bonds are generated, leading to irreversible, tight bonding up to pressures of about 2 bar $[2,6,7]$. The plasma pretreatment is performed in a plasma asher with $275 \mathrm{~W}$ at $13.56 \mathrm{MHz}, 420 \mathrm{sccm} \mathrm{O}$, 2 mbar for $12 \mathrm{~s}$ (Tegal Plasmaline 415). The device is finally bonded to another PDMS layer or to a glass substrate to seal the remaining open channels, utilizing the same plasma process (Fig. 2, 3, 4, 5).

\subsection{Microfluidic chip}

The valves are integrated into a microfluidic chip. The actual chip layout consists of three fluidic inlet channels (e.g. cell solution 1 , cell solution 2 , liquid reagent), one outlet channel and two backflush channels with an inlet and an outlet for both (Fig. 6). Each channel is equipped with a pneumatically actuated valve to open or close the fluid channel (Fig. 7).

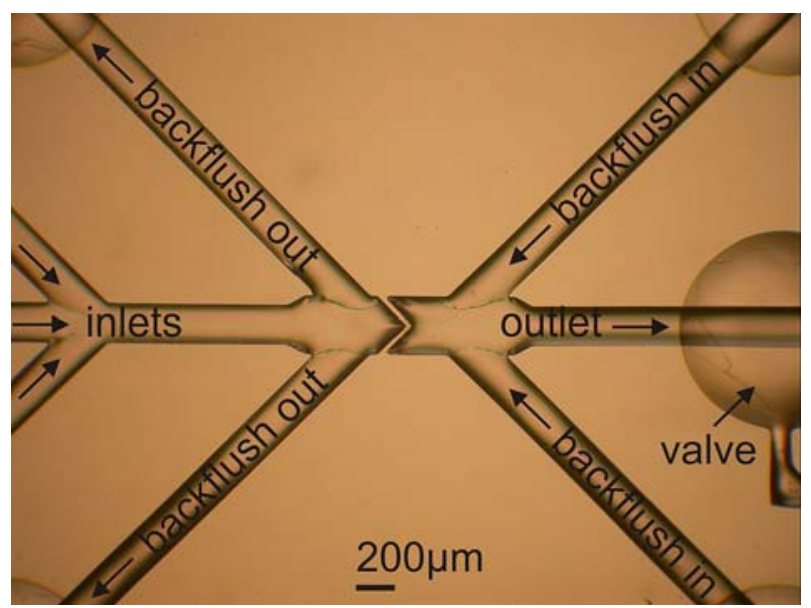

Fig. 6: Microfluidic network with inlet and outlet channels, backflush channels and pneumatic valves

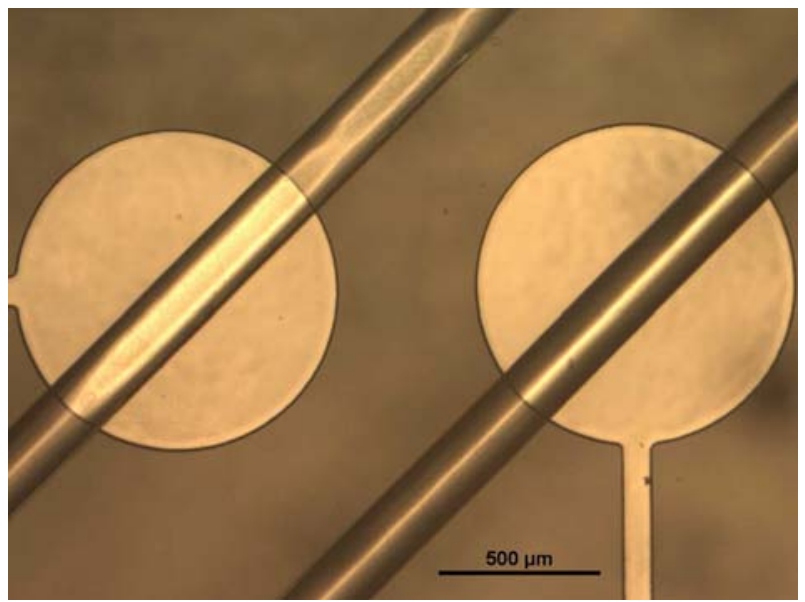

Fig. 7: Pneumatically actuated valves over fluid channels
Two driving mechanisms were investigated for the actuation principle for the cell solution. An actuation mechanism utilizing a peristaltic pump was not feasible due to the low survival rate of the cells due to the mechanical components of the peristaltic pump. Because of these restrictions, a software-controlled pneumatical driving mechanism was developed which actuates the pneumatic layer as well as the fluidic layer. Therefore the whole chip is integrated into an adapter that makes all fluidic and pneumatic channels accessible. A centralized valve group supplied with pressurized air is connected to the adapter. The valve group itself is computer controllable. By using a LABVIEWbased interface, user-defined fluid management is possible (Fig. 8). The reaction chamber itself consists of a beaked structure including a centered passage $5 \mu \mathrm{m}$ in height (Fig. 9). This design is suitable for accumulating cells in the middle of the chamber and bringing them into tight contact. At the same time, it enables a gentle fluid stream, which is necessary for transporting more cell solution or another liquid medium into the chamber.

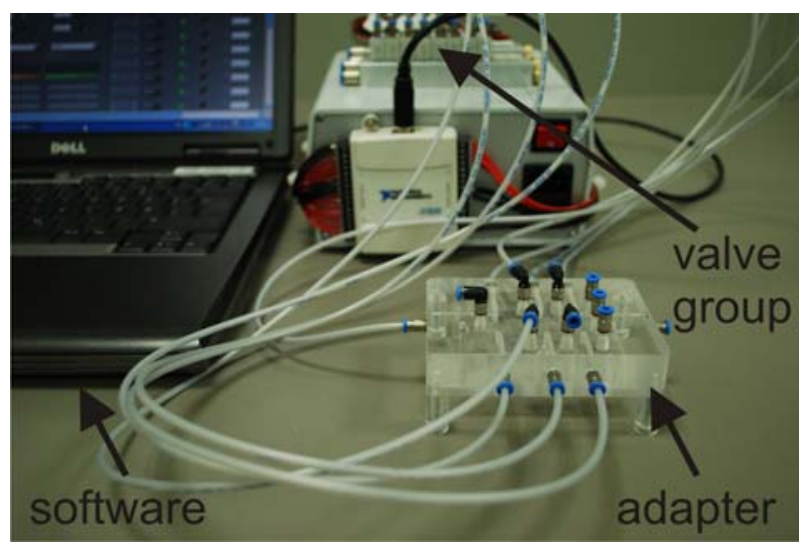

Fig. 8: Experimental setup with a microfluidic chip mounted on an adapter. The adapter is pneumatically connected via a centralized and software-controlled valve group

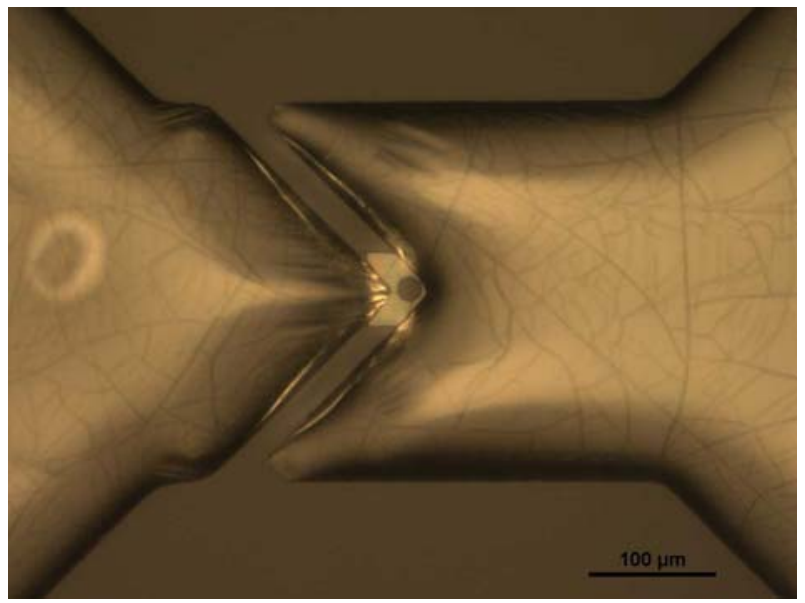

Fig. 9: Reaction chamber. Cells can be accumulated in the central structure 
A typical cell accumulation process consists of the following steps:

1. Open the valve of the exit channel

2. Open the valve of inlet 1 and pump cell solution 1

3. Close the valve of inlet 1

4. Open the valve of inlet 2 and pump cell solution 2

5. Close the valve of inlet 2

6. Open the valve of inlet 3 to flush the accumulated cells with reagent

7. Close the valves of inlet 3 and exit channel

8. Open the valves of one backflush channel and pump the cells out of the chip

\subsection{Cell lines}

In order to analyze the suitability of the microfluidic chip for cell handling, permanent cell lines were identified, which were comparable in size and handling to the final target cells which should be merged within the chip. The human myeloid cell line U937 and the human lymphoid cell line L540 were found to meet these requirements. This avoids laborious preparation of spleen cells from mice and animal consuming procedures for the preliminary experiments. Passages through microfluidic structures may affect cell functions, e.g. by mechanical stress or adherence dependent activation. The viability of the cells after passage of the microfluidic was tested by viability and proliferation assays.

\section{Results}

\subsection{Valve simulations}

All valve concepts were simulated using ANSYS to optimize the geometries. Valve type 1 was at first just operated passively. This means that the pneumatic chamber is not pressurized and the membrane just bends up and down depending on the pressure in the fluidic channel. This concept is optimized by applying a positive or negative pressure to the pneumatic layer. With increasing pressure in the pneumatic chamber in the closed state the bar can be pressed down even more strongly, leading to effective sealing of the fluidic channel that blocks the flow. In the opened state, a relative negative pressure of 0.2 bar in the pneumatic layer results in upward bending of the membrane and the bar, enabling continuous flow. Because the bar is linked at the sides of the channel, increasing negative pressure does not improve the valve opening significantly. With about $15 \mu \mathrm{m}$ in the central region of the valve the opened cross-section of this valve type is clearly smaller than the channel itself, as can be seen in the ANSYS simulations (Fig. 10, 11).

Figures 12, 13 and 14 show the simulation results for valve type 2 . When the pneumatic layer is not pressurized, continuous flow is possible in the fluidic channel and the valve is intrinsically open. In this state,

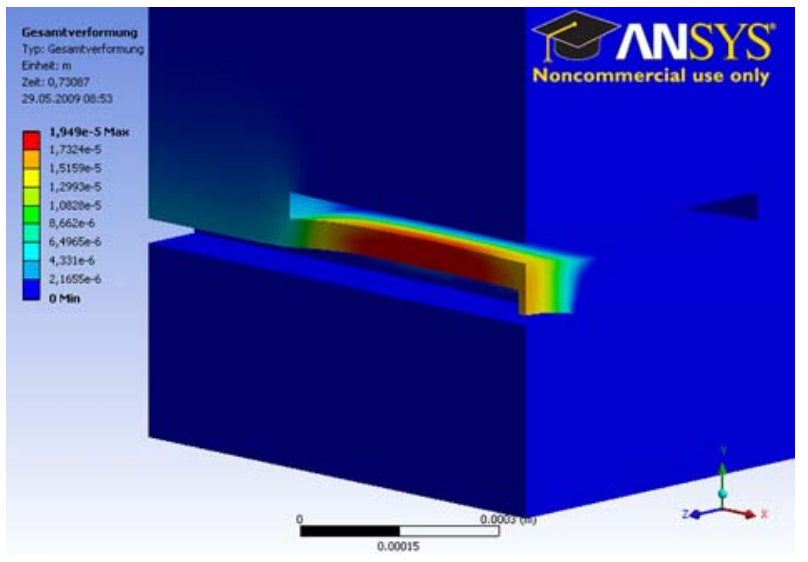

Fig. 10: ANSYS simulation of valve type 1 in the opened state when actuated with a negative pressure of 0.2 bar in the pneumatic layer

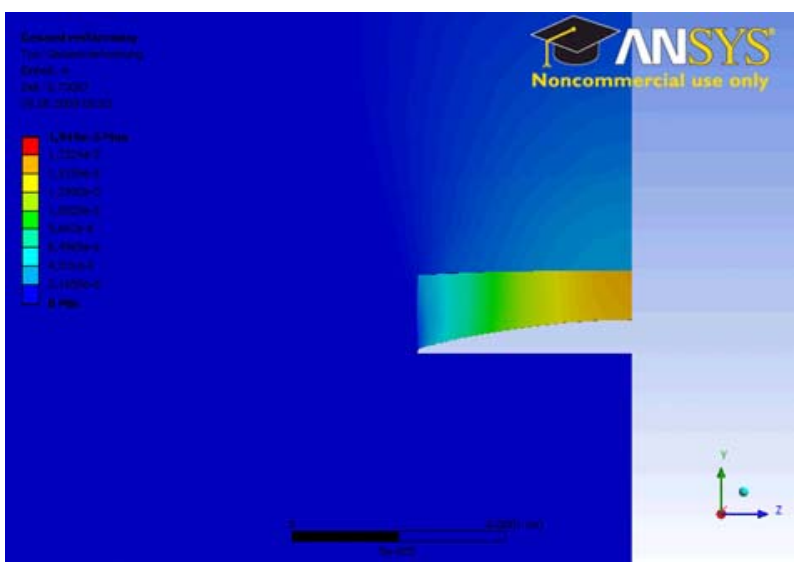

Fig. 11: ANSYS simulation of valve type 1 in the opened state when actuated with negative pressure of 0.2 bar in the pneumatic layer. Perspective from inside the channel

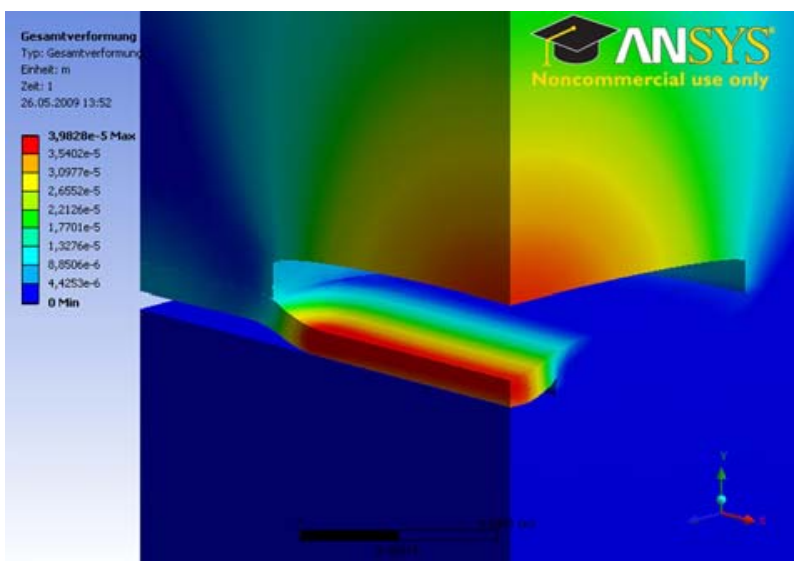

Fig. 12: ANSYS simulation of valve type 2 in the closed state when actuated with 0.5 bar in the pneumatic layer

the opening cross-section of the valve is the same as the channel cross-section itself, allowing undisturbed flow. If pressure is applied to the pneumatic layer, the membrane bends downwards and blocks the channel so that the valve is in the closed state. The ANSYS simulations show insufficient closure of valve type 2 (Fig. 13, 14). 


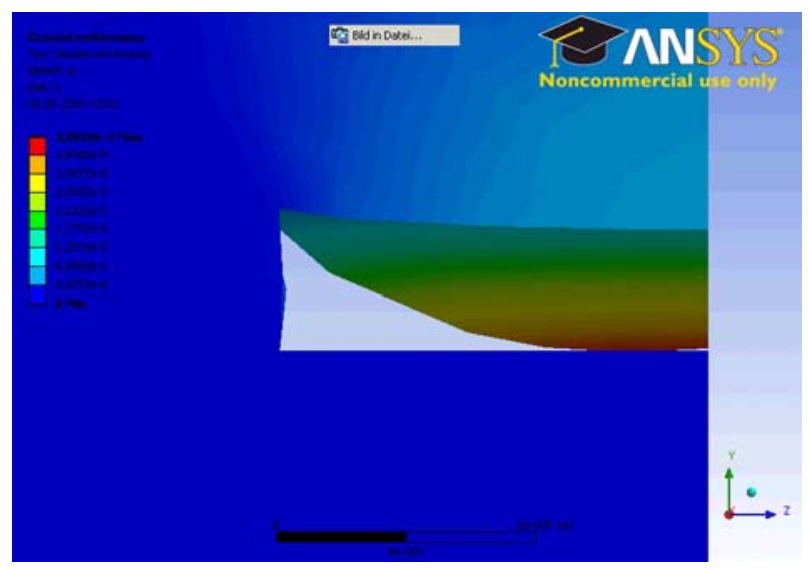

Fig. 13: ANSYS simulation of valve type 2 in the closed state when actuated with 0.5 bar in the pneumatic layer. Perspective from inside the channel

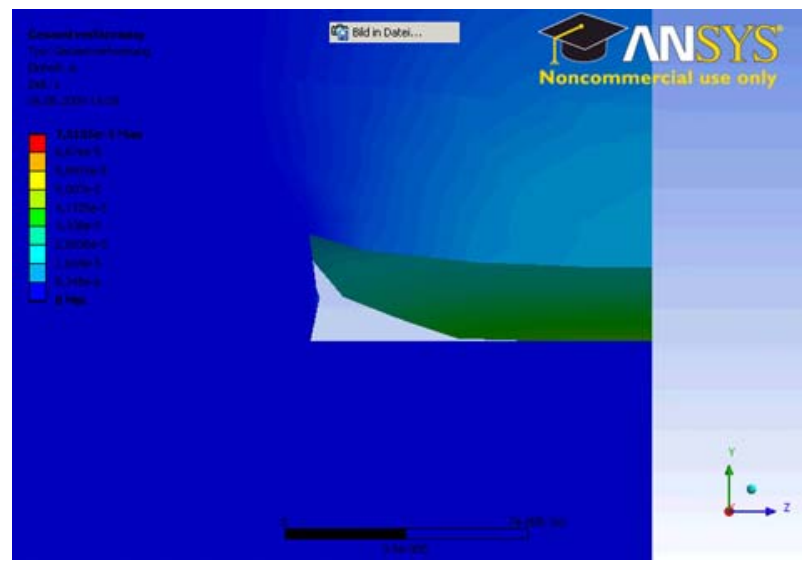

Fig. 14: ANSYS simulation of valve type 2 in the closed state when actuated with 1.0 bar in the pneumatic layer. Perspective from inside the channel

Due to the rectangular fluidic channel, the membrane cannot bent entirely to the bottom of the channel. The simulations show two remaining approximately triangular areas where flow is still possible. In the middle of the channel the closure of the valve is complete. Depending on the pressure applied to the pneumatic layer, a greater or smaller amount of fluid is able to pass the valve. Figure 14 shows that an entire stop to the fluid cannot be obtained within an acceptable pressure range $(<1$ bar $)$. These imperfect closing characteristics also persist if the pressure is increased up to 2 bar. In the same pressure region, valve type 3 shows better sealing than valve type 2 , but no complete flow blockage can be achieved with this modification (Fig. 15).

Figure 16 shows the results of the ANSYS simulations for valve type 4 . This valve type is characterized by a reverse order of the functional layers, as described above. The reverse order results in a membrane which, when actuated by pressure, tightly adapts to the rounded fluidic channel, resulting in re-

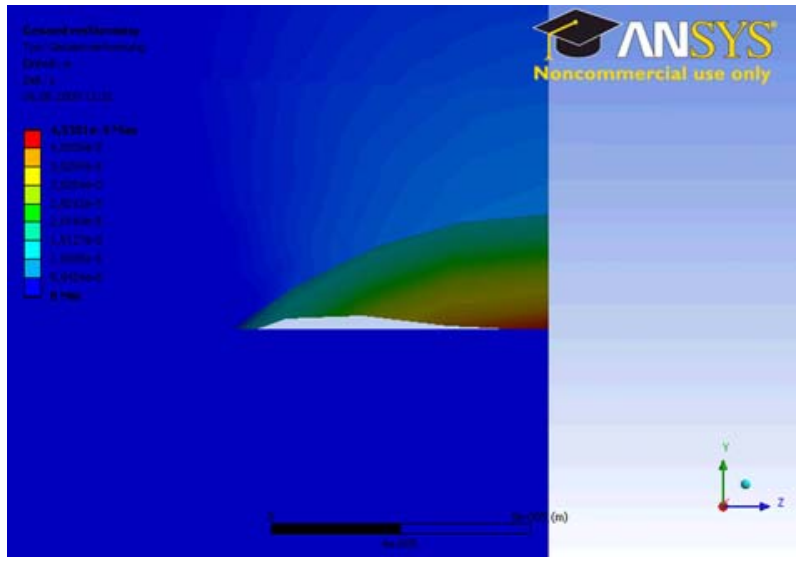

Fig. 15: ANSYS simulation of valve type 3 in the closed state when actuated with 0.5 bar in the pneumatic layer. Perspective from inside the channel

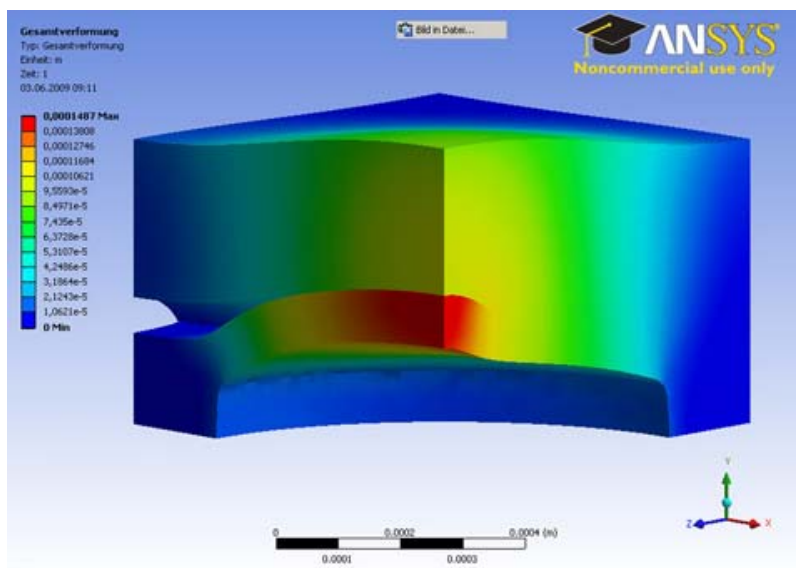

Fig. 16: ANSYS simulation of valve type 4 in the closed state when actuated with 0.75 bar in the pneumatic layer

liable sealing. The ANSYS simulations show effective closure of this valve type for pressures $<1$ bar. In summary, the simulations show that valve types 2 and 3 are not suitable geometries for reliable fluid management. These results were verified in several experimental test series. Valve types 2 and 3 show blocked flow in the middle of the channel. With increasing pressure on the pneumatic layer the closed area of the channel also increases. However, a persistent flowrate is observable in these geometries at the sides of the channel, which cannot be blocked despite an pressure increase of up to 2 bar in the pneumatic layer. By contrast, the simulations indicate that valve types 1 and 4 are adequate designs for a well working valve. Primarily valve type 4 shows excellent properties in the opened state. The cross-section of the valve in this state is the entire cross-section of the fluidic channel. The flow does not need to pass a constriction causing high shear rates, as in type 1 . In the closed state, valve type 4 entirely blocks the flow when a pressure of about $0.75-1$ bar is applied to the pneumatic chamber. In the test series, valve type 4 showed one disadvan- 
tage concerning the integrity of the valve. Due to the stiffness of PDMS, the membrane of the pneumatic channel must not exceed a thickness of about $50 \mu \mathrm{m}$. This is necessary because the membrane is distorted when the valve is actuated and has to apply tightly to the rounded wall of the fluidic channel. An excessively thick membrane limits this distortion and the valve does not entirely block the flow. Due to the low thickness of the membrane, it can be damaged. Careful handling during casting and the whole assembly process is therefore essential. Valve type 1 shows clear advantages concerning the closed state. Its intrinsic closing behavior is already very efficient, and the sealing can be further optimized by a little overpressure in the pneumatic chamber. The disadvantage of valve type 1 is its reduced cross-section in the opened state. When actuated with negative pressure, the membrane and the bar of the valve bend up only in the central region. This produces just a small opening compared to the whole cross-section of the fluidic channel. The test series showed that this is no serious problem when dealing with particle-free solutions. When handling solutions including particles like cells, however, this problem causes obstruction of the valve if the particles are too big to pass the opened part of the channel. This problem makes valve type 1 inapplicable for such applications.

\subsection{Biological tolerance}

The compatibility of the microfluidic chip for cells was tested by viability and proliferation assays to exclude a negative impact of the microfluidic passage on the cells. No significant difference in viability and proliferating activity was observed. The suitability of the different valve designs for cell handling was tested using the human cell lines U937 and L540. Valve type 1 shows excellent results when dealing with cell-free media such as buffer solutions. Unfortunately, cells are generally damaged when passing the valve due to the mechanical stress they are exposed to between the lifted bar and the bottom of the fluidic channel. The test series for valve type 4 showed excellent suitability for cell handling applications. No negative impact on the cell survival rate is caused by this type of valve geometry.

\subsection{Cell accumulation}

In the test series with human cell lines U937 and L540 we showed that the microfluidic chip is suitable for user-defined cell handling. We are able to collect one or more cells within the reaction chamber (Fig. 17). After collecting the cells we can flush them with an arbitrary liquid medium to initiate certain reactions (e.g. cell fusions when using PEG as a medium). Afterwards, the cells can be flushed out of the chip for further biological investigations.

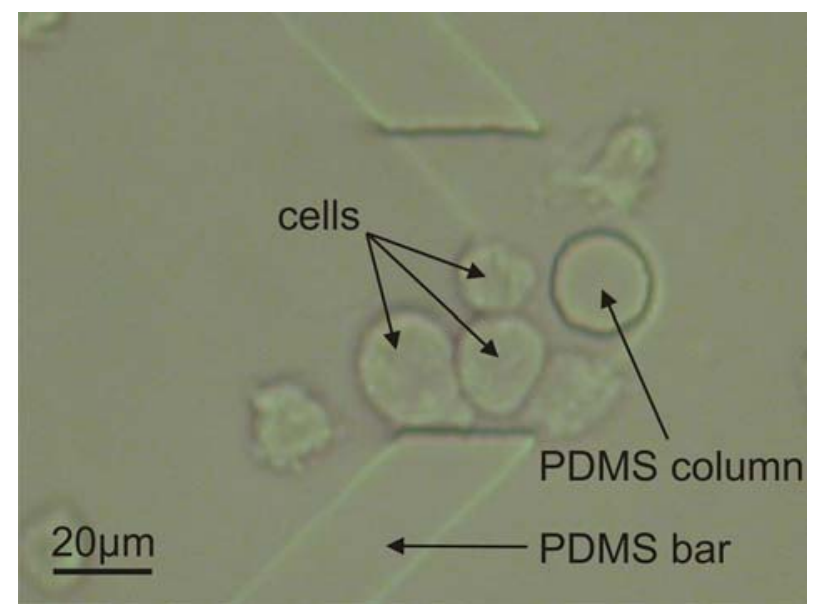

Fig. 17: Cell accumulation in the reaction chamber

\section{Conclusions}

In this paper we have simulated 4 pneumatically actuated valve concepts, and we propose two of them (valve type 1 and 4) as suitable for microfluidic applications in PDMS substrates. The valve function has been simulated in ANSYS and the simulation results have been verified in several test series. One valve type (valve type 4) is shown to be very advantageous when dealing with solutions with particles such as cells inside. The other valve type (valve type 1 ) is proposed for microfluidic applications with particle-free solutions. By integrating these valves into microfluidic channels we designed a pneumatically actuated microfluidic chip for cell handling applications. A software interface allows a user-defined fluid management. The system design is suitable for collecting one or more cells within a reaction chamber, where they can be flushed with a liquid medium to initiate biological reactions such as cell fusion.

\section{Acknowledgement}

The research described in this paper was supervised by Prof. Dr. W. Mokwa, Institute of Materials in Electrical Engineering, RWTH Aachen University, and by Prof. Dr. Dr. S. Barth, Fraunhofer Institute for Molecular Biology, Aachen, and was supported by the Exploratory Research Space RWTH Aachen University under grant No. MSE04.

\section{References}

[1] Cooper McDonald, J., Whitesides, G. M.: Poly(dimethylsiloxane) as a material for fabricating microfluidic devices. Accounts of Chemical Research, 2002, vol. 35, no. 7, p. 491-499.

[2] Cooper McDonald, et al.: Fabrication of microfluidic systems in poly(dimethylsiloxane). Electrophoresis, 2000, vol. 21, p. 27-40. 
[3] Buchenauer, A., et al.: Microbioreactors with microfluidic control and user-friendly connection to the actuator hardware. J. Micromech. Microeng., 2009, vol. 19.

[4] Hosokawa, K., Maeda, R.: A pneumaticallyactuated three-way microvalve fabricated with polydimethylsiloxane using the membrane transfer technique. J. Micromech. Microeng., 2000, vol. 10, p. $415-420$.

[5] Unger, M. A., et al.: Monolithic microfabricated valves and pumps by multilayer soft lithography. Science, 2000, vol. 288, p. 113-116.

[6] Morent, R., et al.: Adhesion enhancement by a dielectric barrier discharge of PDMS used for flexible and stretchable electronics. J. Phys. D: Appl. Phys, 2007, vol. 40, p. 7392-7 401.

[7] Bhattacharya, S., et al.: Studies on surface wettability of poly(dimethyl)siloxane (PDMS) and glass under oxygen-plasma treatment and correlation with bond strength. Journal of Microelectromechanical Systems, 2005, vol. 14, no. 3, p. 590-597.

\section{About the authors}

Mirko GRABOWSKI was born in Bochum, Germany in 1980. He studied electrical engineering at Ruhr-Universität Bochum, focusing on plasma technology. Mirko Grabowski is currently working as a research associate at the Institute of Materials in Electrical Engineering at RWTH Aachen University, Germany, under the supervision of Prof. Dr. W. Mokwa. His work focuses on microfluidic devices for applications in biomedical engineering.

Andreas BUCHENAUER was born in Stuttgart, Germany in 1978. He studied mechanical engineering at RWTH Aachen University, focusing on MEMS technology. Andreas Buchenauer is currently working as a research associate at the Institute of Materials in Electrical Engineering at RWTH Aachen University, Germany, under the supervision of Prof. Dr. W. Mokwa. His work focuses on microfluidic devices for applications in biochemical engineering.

Mirko Grabowski

Andreas Buchenauer

E-mail: grabowski@iwe1.rwth-aachen.de, buchenauer@iwe1.rwth-aachen.de Institute of Materials in Electrical Engineering 1 RWTH Aachen University

Sommerfeldstraße 24, 52074 Aachen, Germany 